\title{
Lymphocele Containing Staphylococcus lugdunensis
}

\author{
Dinesh Keerty ${ }^{1}$, Manoj Das ${ }^{2}$, Timothy N. Hembree ${ }^{1}$, Asha Ramsakal ${ }^{3}$, Elizabeth Haynes ${ }^{1}$ \\ 1. Internal and Hospital Medicine, Moffitt Cancer Center, Tampa, USA 2. Internal Medicine/Nephrology, Geisinger \\ Health System, Danville, USA 3. Internal Medicine, Moffitt Cancer Center, Tampa, USA
}

Corresponding author: Dinesh Keerty, dinesh.keerty@gmail.com

\begin{abstract}
Staphylococcus species are a leading cause of community-acquired bacteremia. Of them, the most serious cause of mortality is from methicillin-resistant Staphylococcus aureus, with mortality rates as high as $40 \%$. Another Staphylococcus species that has been noted to cause equal levels of infection and mortality is Staphylococcus lugdunensis (S. lugdunensis). It can cause harmless skin infections to life-threatening endocardial complications. We would like to report a rare presentation of S. lugdunensis bacteremia from a lymphocele that developed post surgery. An 80-year-old male presented to the emergency department with complaints of abdominal pain and fevers. Cultures of lymphocele fluid grew $\mathrm{S}$. lugdunensis. A computed tomography of the abdomen and pelvis with contrast showed the presence of a large lymphocele. S. lugdunensis is a coagulase-negative staphylococci normally known to be a skin colonizer. Over the years, it has shown to cause a wide variety of infections especially involving prosthetic joints and heart valves. S. lugdunensis has been noted to be highly susceptible to penicillins, such as oxacillin, erythromycin, linezolid and a wide a variety of other antibiotics. S. lugdunensis produces a biofilm that makes treatment challenging even with susceptible antibiotics. However, the data on S. lugdunensis is growing as more case reports are being published in regards to source and susceptibilities.
\end{abstract}

Review began 11/09/2020 Review ended 11/13/2020 Published 11/23/2020

๑) Copyright 2020

Keerty et al. This is an open access article distributed under the terms of the Creative Commons Attribution License CC-BY 4.0., which permits unrestricted use, distribution, and reproduction in any medium, provided the original author and source are credited.
Categories: Internal Medicine, Urology, Infectious Disease

Keywords: coagulase-negative staphylococcus, lymphocele, bacteremia, staphylococcus lugdunesis

\section{Introduction}

Staphylococcus species are a leading cause of community-acquired bacteremia. Of them, the most serious cause of mortality is from methicillin-resistant Staphylococcus aureus, with mortality rates as high as $40 \%$ [1]. Another Staphylococcus species that been noted to cause equal levels of infection and mortality is Staphylococcus lugdunensis (S. lugdunensis). It can cause harmless skin infections to life-threatening endocardial complications [2]. We would like to report a rare presentation of S. lugdunensis bacteremia from a lymphocele that developed post surgery.

\section{Case Presentation}

An 80-year-old male presented to the emergency department with complaints of abdominal pain and fevers. The patient's past medical history is significant for bladder cancer. One month ago, he underwent a radical cystoprostatectomy with ileal conduit placement. Prior to surgery, he was treated with neoadjuvant nivolumab and ipililumab. He stated that over the past two weeks, he had been having abdominal pain and fevers. The fevers were gradual in onset, intermittent, with a max temperature of less than $100.4^{\circ} \mathrm{F}$. Along with the fevers, he complained of left flank and groin pain, aching, which worsened from positional changes. He was taking Tylenol for the pain and fevers for the past two weeks. He denied any signs of hematuria, dysuria, nausea, vomiting, or diarrhea. He denied any recent travels or sick contacts. The patient reported adequate output from the ileal conduit. The patient denied any nausea, vomiting, diarrhea, constipation, or shortness of breath at this time. Vitals were within normal limits and the temperature was $100^{\circ} \mathrm{F}$ on presentation. We performed routine labs, blood cultures, urinalaysis from the ileal conduit, and urine cultures (Table 1). 


\section{Cureus}

\begin{tabular}{|c|c|c|}
\hline LAB TEST & PATIENT'S VALUES & NORMAL \\
\hline White blood cell count & $11.18 \mathrm{k} / \mathrm{uL}$ & $4-10.9 \mathrm{k} / \mathrm{uL}$ \\
\hline Hemoglobin & $11.0 \mathrm{~g} / \mathrm{dL}$ & $13.4-16.9 \mathrm{~g} / \mathrm{dL}$ \\
\hline Platelet count & 193 k/uL & 143-382 k/uL \\
\hline Sodium & $139 \mathrm{mmol} / \mathrm{L}$ & $135-145 \mathrm{mmol} / \mathrm{L}$ \\
\hline Potassium & $4.9 \mathrm{mmol} / \mathrm{L}$ & $3.5-5.0$ mmol/L \\
\hline Chloride & $101 \mathrm{mmol} / \mathrm{L}$ & 96-107 mmol/L \\
\hline Bicarbonate & $25 \mathrm{mmol} / \mathrm{L}$ & $22-30 \mathrm{mmol} / \mathrm{L}$ \\
\hline Urine Nitrite & Positive & Negative \\
\hline Urine Leucocyte Esterase & Moderate & Negative \\
\hline Urine White Blood Cell & $21-50 / \mathrm{hpf}$ & $0-2 / h p f$ \\
\hline Urine Red Blood Cell & 3-5/hpf & $0-2 / h p f$ \\
\hline Urine Bacteria & $1+$ & None \\
\hline
\end{tabular}

TABLE 1: Patient's lab results

A computed tomography of the abdomen and pelvis with contrast showed the presence of a large lymphocele (Figure 1).

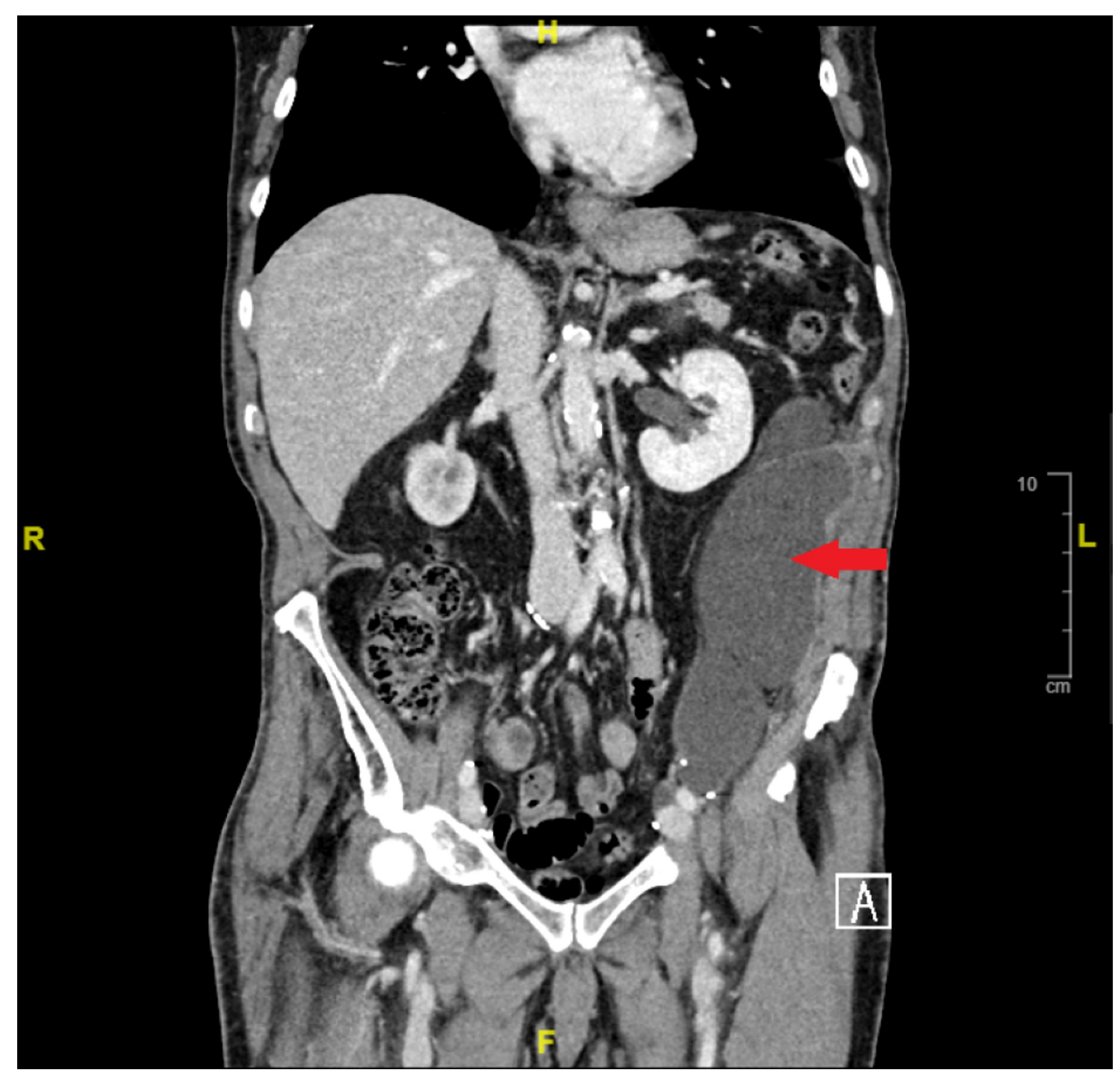

FIGURE 1: Computed tomography of the abdomen and pelvis

Red arrow: Large left flank lymphocele measuring $20.4 \times 9.8 \times 6.2 \mathrm{~cm}$ with no associated gas formation or substantial surrounding inflammation. 
We empirically started him on ceftriaxone. The patient also underwent a drain placement and fluid cultures from the lymphocele were sent. Two days later, blood cultures and fluid cultures showed the presence of S. lugdunensis with susceptibilities (Table 2).

\begin{tabular}{|c|c|c|}
\hline \multicolumn{3}{|c|}{ Staphylococcus lugdunensis } \\
\hline & Sensitivity & Minimum inhibitory concentration \\
\hline Penicillin & $\mathrm{R}$ & \\
\hline Ciprofloxacin & $\mathrm{s}$ & $<=0.5$ \\
\hline Clindamycin & $\mathrm{s}$ & $<=0.25$ \\
\hline Tetracycline & $\mathrm{S}$ & $<=1$ \\
\hline Trimethoprim/Sulfa & $\mathrm{s}$ & $<=10$ \\
\hline Vancomycin & $S$ & $<=0.5$ \\
\hline
\end{tabular}

TABLE 2: Staphylococcus lugdunensis susceptibilities from blood and fluid cultures

Ceftriaxone was stopped and vancomycin was started. Patient was subsequently discharged two days later with 2-week course of intravenous vancomycin. Patient had resolution of his symptoms after two weeks and had negative blood cultures.

\section{Discussion}

S. lugdunensis is a coagulase-negative staphylococci normally known to be a skin colonizer [3]. Over the years, it has shown to cause a wide variety of infections especially involving prosthetic joints and heart valves [4-5]. Bocher et al. noted that majority of the cases were found in skin abscesses, wound infections, and paronychias [3]. However, serious infections could occur from S. lugdunensis affecting prosthetic joints and heart valves. Patients infected by S. lugdunensis have shown to have concurrent comorbidities such as chronic immunosuppression, diabetes, or recent trauma [4-6]. Skin infections and abscess formations are the most common sources of S. lugdunensis infections [3]. S. lugdunensis has been noted to demonstrate antibiotic sensitivity similar to methicillin-sensitive Staphylococcus aureus [5].

When the diagnosis of S. lugdunensis occurs, there is difficulty in determining if it is a clinically significant infection or from contamination of the specimen or colonization of skin or mucous membranes [7]. Therefore repeat cultures are often recommended before S. lugdunensis infection can be diagnosed [8]. The use of matrix-assisted laser desorption/ionization time of flight mass spectrometry (MALDI-TOF MS) in laboratories has resulted in more accurate S. lugdunensis identification [9].

S. lugdunensis has been noted to be highly susceptible to penicillins, such as oxacillin, erythromycin, linezolid, and a wide variety of other antibiotics [3-4]. Bocher et al. state they noted no difference in antimicrobial susceptibility between isolates from hospitalized patients and isolates from general practice. S. lugdunensis produces a biofilm that makes treatment challenging even with susceptible antibiotics [3]. Such is the case with our patient, where it was resistant to penicillin. We, therefore, utilized vancomycin to be more effective. However, the data on S. lugdunensis is growing as more case reports are being published in regards to source and susceptibilities.

\section{Conclusions}

S. lugdunensis is common skin commnesal that can result in significant clinically relevant infections when noted in abscess, heart valves, or prosthetic joints. It should be diagnosed with repeat cultures and MALDITOF MS in clinical laboratories. As we gain more knowledge of its susceptibilities and infectivity patterns, we can improve upon our treatment of S. lugdunensis.

\section{Additional Information}

\section{Disclosures}

Human subjects: Consent was obtained by all participants in this study. Conflicts of interest: In compliance with the ICMJE uniform disclosure form, all authors declare the following: Payment/services info: All authors have declared that no financial support was received from any organization for the 
submitted work. Financial relationships: All authors have declared that they have no financial relationships at present or within the previous three years with any organizations that might have an interest in the submitted work. Other relationships: All authors have declared that there are no other relationships or activities that could appear to have influenced the submitted work.

\section{References}

1. Mylotte JM, McDermott C, Spooner JA: Prospective study of 114 consecutive episodes of Staphylococcus aureus bacteremia. Rev Infect Dis. 1987, 9:891-907. 10.1093/clinids/9.5.891

2. Frank KL, del Pozo JL, Patel R: From clinical microbiology to infection pathogenesis: how daring to be different works for Staphylococcus lugdunensis. Clin Microbiol Rev. 2008, 21:111-33. 10.1128/CMR.0003607

3. Bocher S, Tonning B, Skov RL, Prag J: Staphylococcus lugdunensis, a common cause of skin and soft tissue infections in the community. J Clin Microbiol. 2009, 47:946-950. 10.1128/JCM.01024-08

4. Parthasarathy S, Shah S, Raja Sager A, Rangan A, Durugu S: Staphylococcus lugdunensis: review of epidemiology, complications, and treatment. Cureus. 2020, 12:e8801. 10.7759/cureus.8801

5. Heldt Manica LA, Cohen PR: Staphylococcus lugdunensis infections of the skin and soft tissue: a case series and review. Dermatol Ther. 2017, 7:555-562. 10.1007/s13555-017-0202-5

6. Herchline TE, Ayers LW: Occurrence of Staphylococcus lugdunensis consecutive clinical cultures and relationship of isolation to infection. J Clin Microbiol. 1991, 29:419-421. 10.1128/JCM.29.3.419-421.1991

7. Becker K, Heilmann C, Peters G: Coagulase-negative staphylococci. Clin Microbiol Rev. 2014, 27:870-926. 10.1128/CMR.00109-13

8. Natsis NE, Cohen PR: Coagulase-negative Staphylococcus skin and soft tissue infections . Am J Clin Dermatol. 2018, 19:671-677. 10.1007/s40257-018-0362-9

9. Argemi X, Hansmann Y, Riegel P, Prévost G: Is Staphylococcus lugdunensis significant in clinical samples? . J Clin Microbiol. 2017, 55:3167-3174. 10.1128/JCM.00846-17 